BIOCYT Biología, Ciencia y Tecnología, 12(46): 855-868, 2019 (abril-junio)

ISSN: 2007-2082

http://revistas.unam.mx/index.php/biocyt

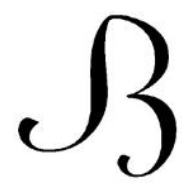

Publicada en la Facultad de Estudios Superiores Iztacala, Universidad Nacional Autónoma de México

\title{
EFECTO DEL ÁCIDO INDOL-3-BUTÍRICO SOBRE LA CALIDAD POSCOSECHA DE GRANADA ROJA (PUNICA GRANATUM L.) 'APASEO TARDÍA'
}

\section{EFFECT OF INDOLE-3-BUTYRIC ACID ON THE POSTHARVEST QUALITY OF POMEGRANATE (PUNICA GRANATUM L.) 'APASEO TARDÍA'}

\author{
Celia Emma Solís-Guzmán ${ }^{1,1}$, Ismael Aguilar-Ayala1,2》 y María Graciela Molina González ${ }^{1,3}$ \\ 1Facultad de Estudios Superiores Iztacala. Universidad Nacional Autónoma de México (UNAM). Avenida de \\ los Barrios Número 1, Los Reyes Iztacala, Tlalnepantla, Estado de México, México. C.P. 54090. \\ 1,1ㄹmmasolis225@gmail.com,1,2》 biofrut94@gmail.com,1,3
}

\begin{abstract}
The concentration of the production of the fruit of the red pomegranate 'Apaseo Tardia' takes place in a period of approximately 40 days; what refers to the economy of its producers to have to bid their production at low prices. On the other hand, the application of phytohormones to fruit after harvest, such as auxins, can be retained with characteristics of adequate quality for long periods of time and in a way that is affordable for users. This allows commercializing the fruits of the red pomegranate outside the harvest period, increasing the sale prices. This research was conducted to evaluate the effect of indole-3-butyric acid (IBA) on postharvest fruit quality red pomegranate fruit (Punica granatum) 'A paseo Tardia'. The treatments evaluated were: 0, 20, 40 and $80 \mathrm{mg} / \mathrm{L}^{-1}$ of IBA. The experimental unit consisted of a fruit with five repetitions for the treatment and the experimental design was completely random. The application of IBA was performed by immersion for 10 minutes. The response variables evaluated were mass $(\mathrm{g})$, size $(\mathrm{cm})$ in fruit diameter and length, total anthocyanin concentration, percentage of titratable acidity, concentration of ascorbic acid, chlorophyll $a, b$ and total. The results indicate that the treatment of $20 \mathrm{mg} / \mathrm{L}^{-1}$ of IBA also conserves the size in length of the fruit. The differences in the statistics in any of the treatments are not found in the other variables evaluated.
\end{abstract}

Key words: auxins, chemicophysical growth, postharvest physiology, prolongation of consumption maturity, regulator properties, shelf life, storage.

Manuscrito recibido el 24 de octubre de 2017, aceptado el 29 de abril de 2019. 


\section{RESUMEN}

La concentración de la producción del fruto de granada roja 'Apaseo Tardía' ocurre en un periodo aproximado de 40 días; lo que afecta la economía de sus productores al tener que ofertar su producción a bajos precios. Por otra parte, la aplicación de fitohormonas al fruto después de la cosecha, como las auxinas, puede conservarlo con características de calidad adecuada por periodos de tiempo prolongado y de forma asequible para los consumidores. Esto permitiría comercializar los frutos de la granada roja fuera del periodo de cosecha, incrementando los precios de venta. La presente investigación se realizó con el objetivo de evaluar el efecto del ácido indol-3-butirico (AIB) sobre la calidad poscosecha del fruto de granada roja (Punica granatum) 'Apaseo Tardía'. Los tratamientos evaluados fueron: 0, 20, 40 y $80 \mathrm{mg} / \mathrm{L}^{-1}$ de AIB. La unidad experimental consistió de un fruto con cinco repeticiones por tratamiento y el diseño experimental fue completamente al azar. La aplicación de AIB se realizó por inmersión durante 10 minutos. Las variables de respuesta evaluadas fueron masa $(\mathrm{g})$, tamaño $(\mathrm{cm})$ en diámetro y longitud del fruto, concentración de antocianinas totales, porcentaje de acidez titulable, concentración de ácido ascórbico, clorofilas $a, b$ y total. Los resultados indican que el tratamiento de $20 \mathrm{mg} / \mathrm{L}^{-1}$ de AIB conservo significativamente el tamaño en longitud del fruto. En las otras variables evaluadas no se encontraron diferencias estadísticas significativas en ninguno de los tratamientos.

Palabras clave: almacenamiento, auxinas, fisiología poscosecha, prolongación de madurez de consumo, propiedades fisicoquímicas, reguladores de crecimiento, vida en anaquel.

\section{INTRODUCCIÓN}

La granada roja (Punica granatum L. 1753) es un frutal nativo de Persia (Verma et al., 2010) que actualmente es cultivado en regiones con climas tropicales y subtropicales de todo el mundo, la capacidad que tiene esta planta para desarrollarse en diferentes condiciones climáticas indica su flexibilidad, adaptabilidad y su amplia diversidad genética (Frison y Servisking, 1995), misma que ha sido demostrada por las más de 500 variedades distribuidas en todo el mundo, aunque solamente 50 de ellas se cultivan de manera comercial (IPGRI, 2001).

Esta planta con importancia económica ha sido utilizada desde las civilizaciones ancestrales debido a su gran potencial de beneficios para la salud (Cohen, 2011) como lo indican investigaciones recientes en las que se reportan propiedades nutritivas, farmacéuticas, y prebióticas, esta última característica se genera en conjunto con otras plantas (Aviram y Dornfeld, 2001; Borochov-Neori et al., 2009; López-Mejía et al., 2010; Peñalver et al., 2011) o su importancia para tratar la obesidad y la diabetes, entre otras enfermedades (Al-Muammar y Khan, 2012).

Debido a la importancia ancestral y actual de la granada roja, su superficie de cultivo ha aumentado siendo ampliamente producida en España, Marruecos, Egipto, Afganistán y Balauchistán, a éstos esfuerzos se han sumado, aunque en pequeña escala, Birmania, Palestina, Pakistán, India, China, Israel, Japón, EUA y México (Mir et al., 2012). En México, Guanajuato e Hidalgo son los estados productores de granada roja con fines comerciales, aunque, la superficie cultivada no se ha determinado con exactitud (López-Mejía et al., 2010). Con relación a esto, el Valle del Mezquital, estado de Hidalgo, durante los últimos años ha contribuido a la exportación de granada roja para países de la Unión Europea y a EUA (SAGARPA, 2015). 
La comercialización de la granada roja, como todo producto hortofrutícola está limitada por su calidad, siendo los factores agroecológicos, manejo precosecha y poscosecha del cultivo quienes la determinan (Demerutis, 2010).

En este sentido, los frutos son órganos vivos sujetos a cambios fisiológicos y bioquímicos continuos, es decir, aún después de ser cosechados continúan respirando, madurando en algunos casos e iniciando procesos de senescencia, todo esto conlleva a una serie de cambios estructurales, biológicos, químicos y de componentes que son específicos para cada fruta (Arias y Toledo, 2000), por lo que durante el manejo poscosecha se pretenden evitar daños físicos y mecánicos, mantener la humedad adecuada para evitar pérdidas de masa por transpiración del fruto y retardar los cambios bioquímicos para prolongar la calidad del fruto en almacén por periodos prolongados (Demerutis, 2010), de tal forma que el manejo adecuado del fruto en poscosecha desempeña una función importante en la actividad comercial de los frutos (El-Ramady et al., 2015).

Por otra parte, la cosecha y comercialización del fruto de granada roja específicamente el cultivar Apaseo tardía en los estados productores de México es de un periodo de 30 a 40 días, ocasionando una sobre oferta del fruto y por lo tanto bajos precios que afectan fuertemente los ingresos económicos de los productores. Ante esta situación una probable solución es almacenar los frutos utilizando diversas técnicas como las atmósferas controladas y modificadas, mejoramiento genético, conservación de la maduración con frío o altas temperaturas e incluso la combinación de algunas de éstas técnicas. Otra forma de conservar los frutos en poscosecha es con la aplicación de reguladores de crecimiento, mismos que se utilizan comúnmente para alterar la fisiología de los tejidos, órganos o toda la planta en general, retrasando o induciendo la senescencia (Kader, 2002).

En cítricos se utilizan giberelinas para retrasar el cambio en el metabolismo de los pigmentos, que está relacionado con la senescencia del pericarpio (Goldschmidt, 1980). En manzanas 'Brookfield' la aminoetoxivinilglicina conserva la calidad del fruto durante el almacenamiento (Brackmann et al., 2015), éste mismo regulador de crecimiento mantiene la firmeza y el color en ciruelas 'Tegan Blue' (Jobling et al., 2003). En uvas de mesa y kiwis se han empleado las citocininas para mantener el calibre y el peso de los frutos (Reynolds et al., 1992). El etileno es utilizado para provocar la desverdización en los frutos no climatéricos, como naranjas, cerezas, manzanas y algunas variedades de chile (Salveit, 1999). El retraso de la senescencia en fresas chilenas se ha logrado a partir de la aplicación de ácido indolacético (AIA), una auxina (Figueroa et al., 2012).

Las auxinas son hormonas vegetales encargadas de regular muchos aspectos del crecimiento y desarrollo de las plantas, tejidos y órganos, como los frutos, debido a que inducen división, crecimiento y diferenciación celular (Jordán y Casaretto, 2006). El ácido indol-3-butírico (AIB), inicialmente, fue clasificado como una auxina sintética, pero es un compuesto endógeno de la planta, más eficiente que el AIA (Jordán y Casaretto, 2006).

No se han reportado estudios en los que se analice el efecto del AIB en fisiología poscosecha, sin embargo, al inducir división celular se espera que pueda retardar la senescencia en frutos de granada roja Apaseo tardía para prolongar su vida en anaquel; ya que la senescencia es la etapa final del proceso de desarrollo del fruto que va de la madurez fisiológica, hasta la completa y final perdida de organización y función del órgano o de todo el organismo para alcanzar la muerte (Bidwell, 1993). 
Por lo anterior, la presente investigación tuvo como objetivo evaluar el efecto del ácido indol-3-butírico sobre la calidad poscosecha de granada roja ( $P$. granatum) 'Apaseo tardía', con la finalidad de prolongar la vida en anaquel de los frutos bajo condiciones de almacén y que conserven características de calidad para su consumo.

\section{MATERIALES Y MÉTODOS}

El material experimental fue obtenido de una huerta comercial establecida con granada roja 'Apaseo tardía' en Ixmiquilpan, Hidalgo, México. Los frutos se cosecharon en la segunda semana del mes de septiembre de 2015 en madurez de consumo con un tamaño promedio de $5.5 \mathrm{~cm}$ de longitud y $5 \mathrm{~cm}$ de diámetro, color uniforme, sin defectos físicos y sin ataque de plagas y enfermedades. Posteriormente, se trasladaron al laboratorio de morfofisiología vegetal de la Facultad de Estudios Superiores Iztacala, UNAM, para ser almacenados en refrigeración a $4 \pm 1{ }^{\circ} \mathrm{C}$ por 24 h, después se lavaron en una solución de hipoclorito de sodio al $5 \%$.

El diseño experimental consistió de cuatro tratamientos: 0, 20, 40 y $80 \mathrm{mg} / \mathrm{L}^{-1}$ de ácido indol-3-butírico, la unidad experimental fue un fruto con cinco repeticiones y el diseño experimental utilizado fue completamente al azar. Los tratamientos se aplicaron por inmersión por un tiempo de 10 minutos y se almacenaron durante 60 días a $4 \pm 1^{\circ} \mathrm{C}$.

Las variables de respuesta se evaluaron de la siguiente manera: Para la masa (g), se utilizó una balanza granataria; el tamaño $(\mathrm{cm})$ en longitud y diámetro del fruto, se midió con una regla vernier. Se realizó una sustracción de la masa y el tamaño en longitud y diámetro del fruto inicial menos los valores finales de estas variables.

Para evaluar la concentración de antocianinas totales se utilizó la técnica propuesta por Kannangara y Hansson (1998) con la siguiente modificación: a una muestra de $0.5 \mathrm{ml}$ de jugo se le adicionaron $10 \mathrm{ml}$ de $\mathrm{HCl} 0.1 \mathrm{~N}$ y las muestras se leyeron a una longitud de onda de $516 \mathrm{~nm}$ con espectrofotómetro marca Shimadzu UV_1201S. En el cálculo del porcentaje de acidez titulable se usó la técnica de la Asociation Oficial Analisys Chemical (AOAC, 1992), tomando $50 \mathrm{~mL}$ de jugo para obtener una alícuota de $20 \mathrm{ml}$ que se tituló con $\mathrm{NaOH}$ al $0.1 \mathrm{~N}$ llevándola a un pH de 8.1-8.3 con un potenciómetro marca Hanna; para realizar los cálculos del porcentaje de acidez titulable se utilizó el ácido málico, que es el ácido de mayor concentración en el jugo de la granada roja.

La determinación de la concentración de ácido ascórbico en el jugo de la granada se realizó con la técnica descrita por Jagota y Dani (1982), para lo cual se realizó una curva patrón lineal 5-70 $\mu \mathrm{g} / \mathrm{mL}$ de ácido ascórbico. La muestra que se tomó fue de $0.5 \mathrm{~mL}$ de jugo y las muestras se leyeron con un espectrofotómetro marca Shimadzu UV_1201S a una longitud de onda de $760 \mathrm{~nm}$.

La cuantificación de clorofila total, $a$ y $b$ se realizó con la técnica propuesta por Witham et al. (1971), las muestras se obtuvieron del pericarpio del fruto para lo cual se utilizó un rayador para obtener trozos pequeños y finos, que posteriormente fueron mezclados. La muestra que se utilizo fue de $500 \mathrm{mg}$ de cada repetición. Las muestras se leyeron a una longitud de onda de 645 y de 663 $\mathrm{nm}$, para clorofila $a \mathrm{y} b$ respectivamente. Los datos se reportaron en $\mathrm{mg} / \mathrm{g}$ de clorofila total, $a$ y $b$.

Los resultados obtenidos en todas las variables fueron analizados mediante un ANOVA. Se realizó la prueba de Tukey $(\alpha=0.05)$ para comparar las medias. 


\section{RESULTADOS}

El tratamiento de $20 \mathrm{mg} / \mathrm{L}^{-1}$ de AIB conservó significativamente el tamaño en longitud del fruto con $0.64 \mathrm{~cm}$ en comparación con el tratamiento testigo, que tuvo una pérdida de $1.42 \mathrm{~cm}$ (Fig.1). Por otro lado, los resultados de masa y diámetro obtenidos indican que no se encontraron diferencias estadísticas significativas (Figs. 2 y 3 ).

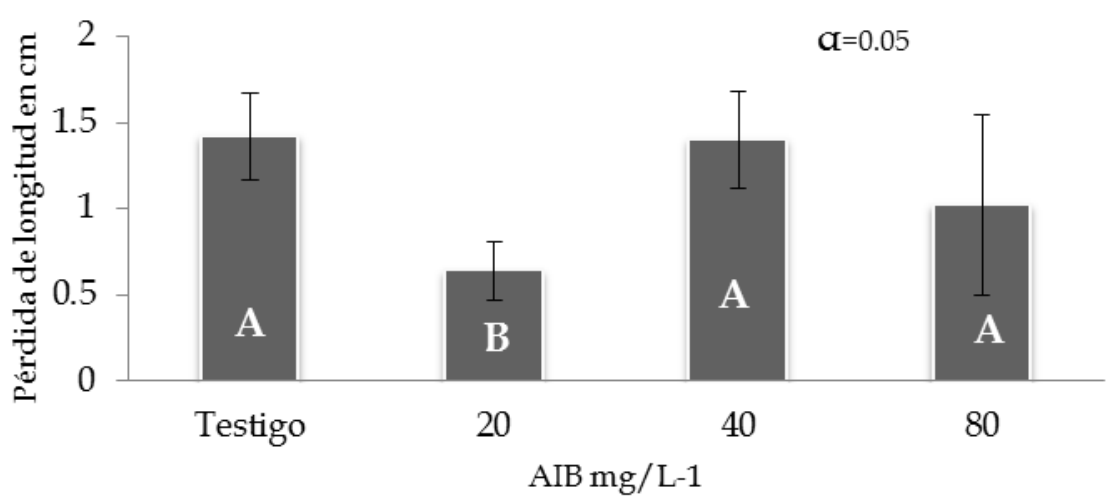

Fig. 1. Efecto del AIB sobre la longitud del fruto de granada roja 'Apaseo tardía'. Las letras iguales indican que no existen diferencias significativas entre tratamientos.

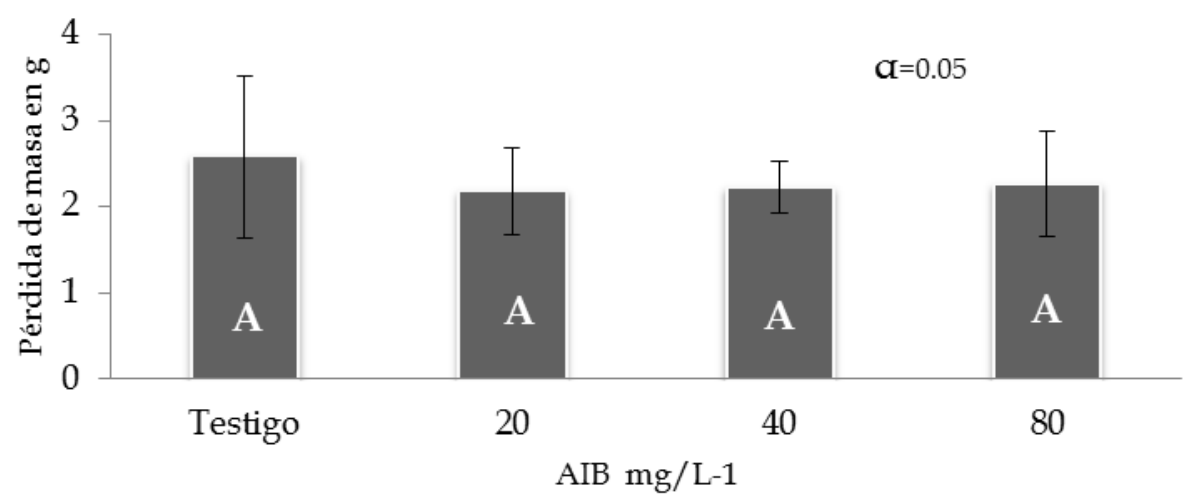

Fig. 2. Efecto del AIB sobre la masa del fruto de granada roja 'Apaseo tardía'. Las letras iguales indican que no hay diferencias significativas entre tratamientos. 


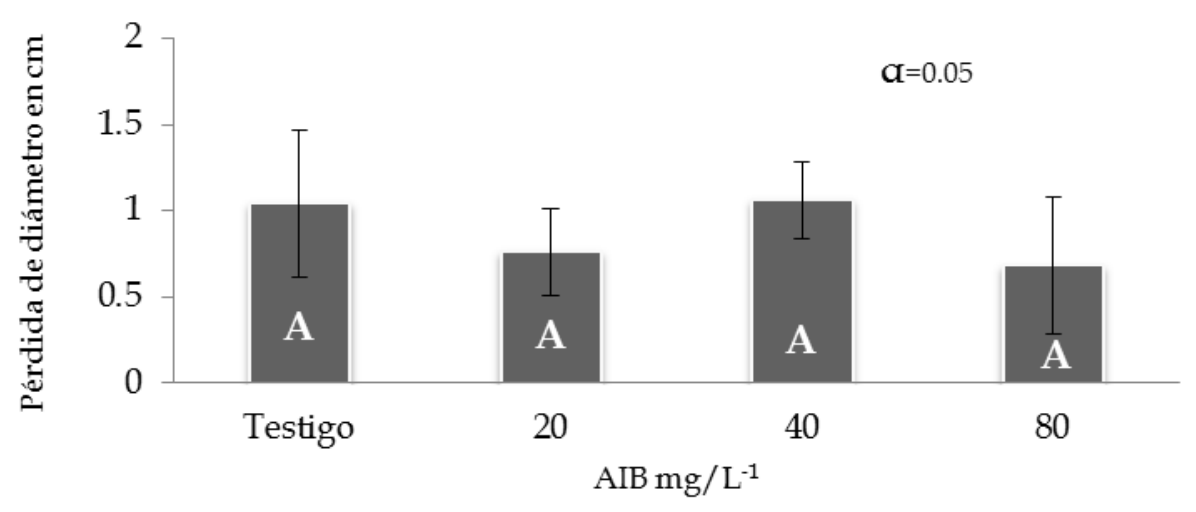

Fig. 3. Efecto del AIB sobre el diámetro del fruto de granada roja 'Apaseo tardía'. Las letras iguales indican que no hay diferencias significativas entre tratamientos.

En cuanto a la concentración de antocianinas totales presentes en el jugo de granada no se encontraron diferencias estadísticas significativas. Sin embargo, el tratamiento de $80 \mathrm{mg} / \mathrm{L}^{-1}$ de AIB presentó una concentración mayor en comparación con el tratamiento testigo, obteniendo 141.96 y $124.11 \mathrm{mg} / 100 \mathrm{ml}$ de jugo, respectivamente (Fig. 4).

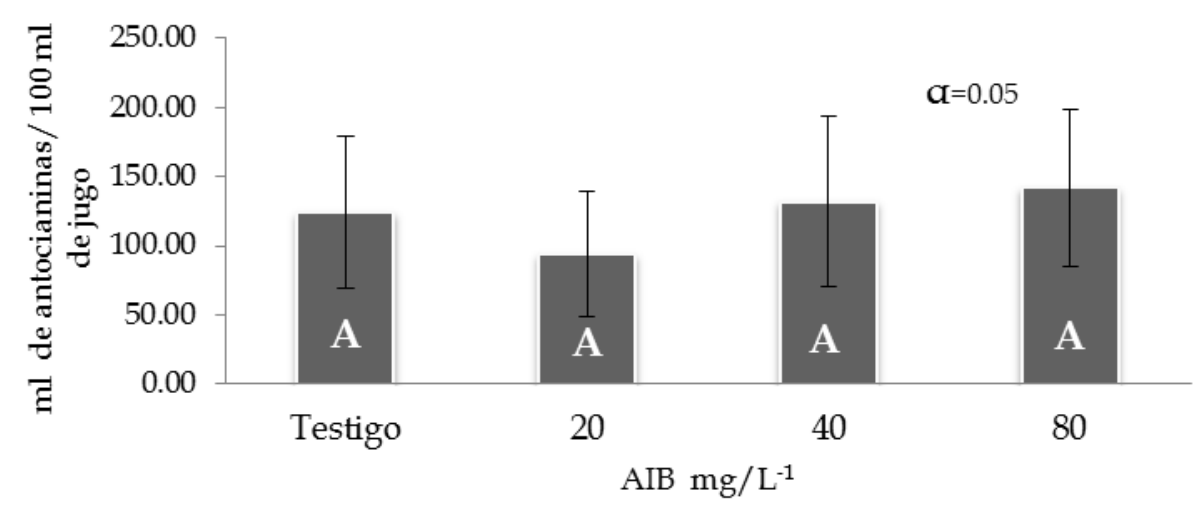

Fig. 1. Efecto del AIB sobre la concentración de antocianinas totales en el fruto de granada roja 'Apaseo tardía'. Las letras iguales indican que no hay diferencias significativas entre tratamientos.

Respecto al porcentaje de acidez titulable del jugo de $P$. granatum no se presentaron diferencias estadísticas significativas entre tratamientos (Fig. 5). 


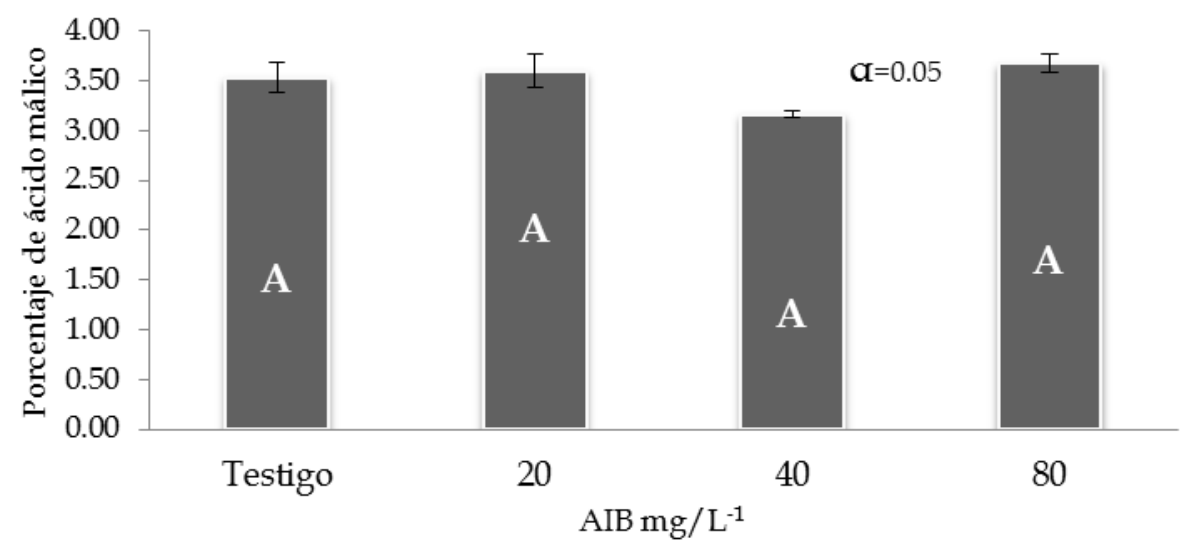

Fig. 2. Efecto del AIB sobre el porcentaje de ácido málico en el fruto de granada roja 'Apaseo tardía'. Las letras iguales indican que no hay diferencias significativas entre tratamientos.

De la misma forma, la concentración de ácido ascórbico no presentó diferencias significativas entre los tratamientos evaluados, pero el tratamiento de $80 \mathrm{mg} / \mathrm{L}^{-1}$ de AIB tuvo mayor concentración con respecto al tratamiento testigo, obteniendo respectivamente 43.90 y $37.51 \mathrm{mg}$ de ácido ascórbico/ $100 \mathrm{ml}$ de jugo (Fig. 6).

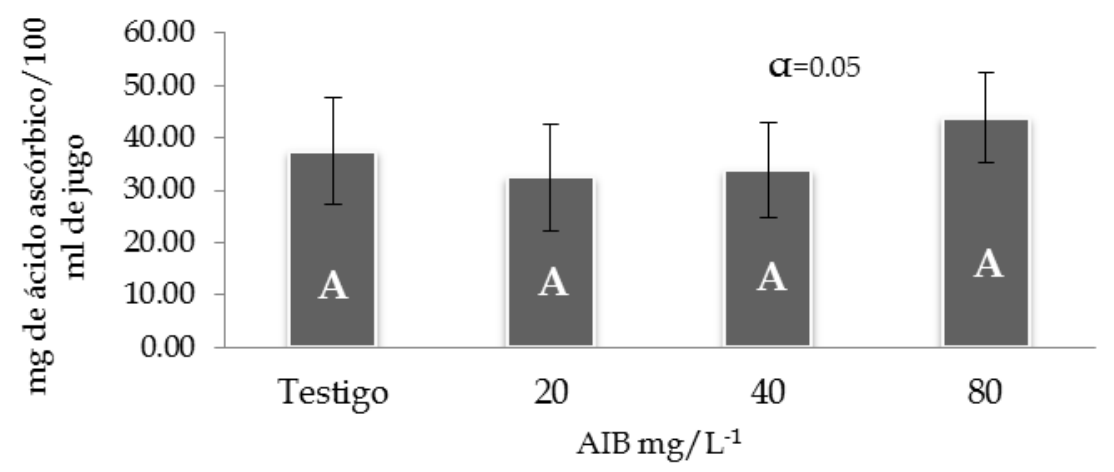

Fig. 3. Efecto del AIB sobre la concentración de ácido ascórbico en el fruto de granada roja 'Apaseo tardía'. Las letras iguales indican que no hay diferencias significativas entre tratamientos.

Asimismo, las concentraciones de clorofila total, $a$ y $b$ tampoco presentaron diferencias estadísticas significativas para los tratamientos aplicados (Figs. 7, 8 y 9). 


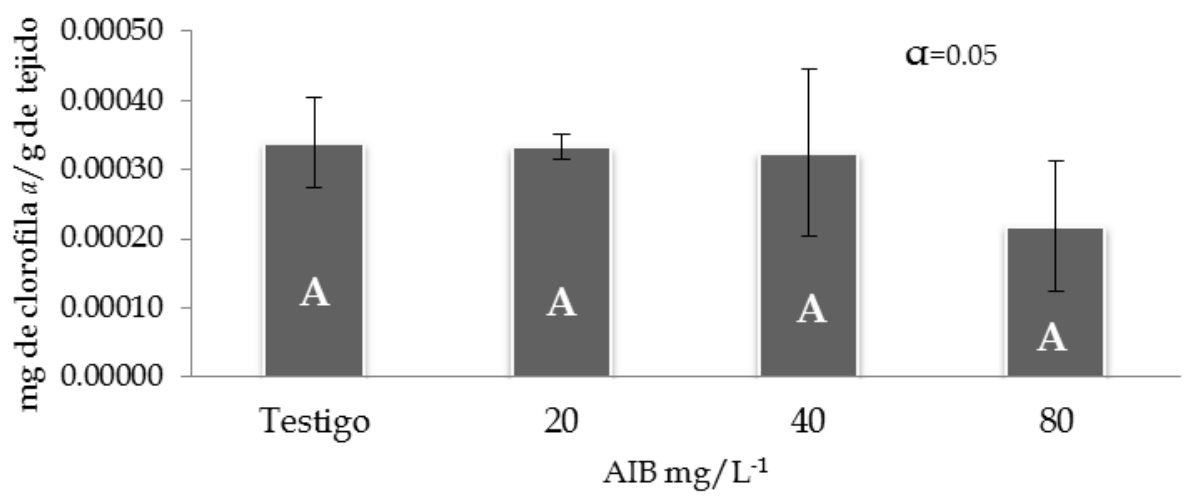

Fig. 4. Efecto del AIB sobre la concentración de clorofila a en el fruto de granada roja 'Apaseo tardía'. Las letras iguales indican que no hay diferencias significativas entre tratamientos.

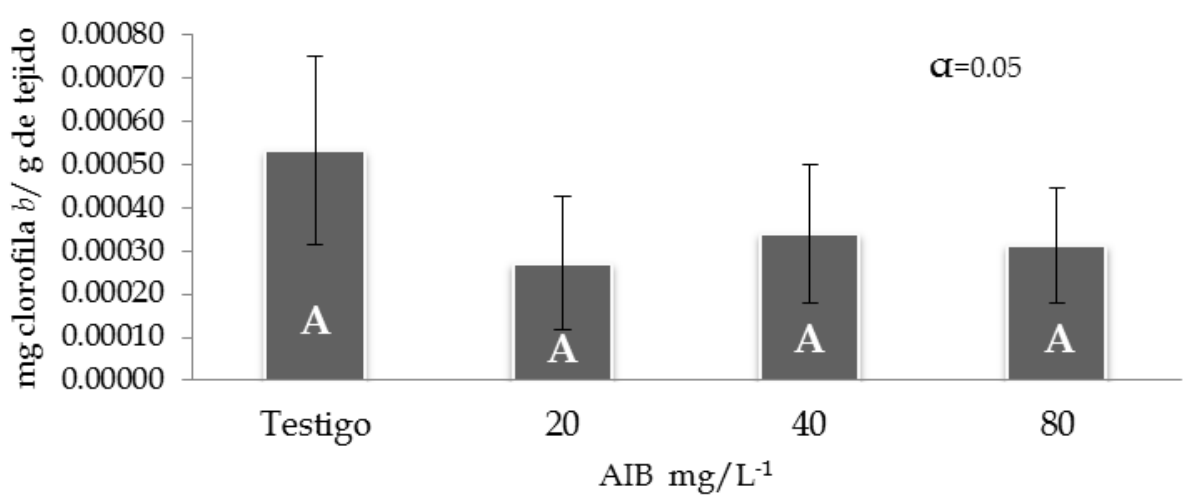

Fig. 5. Efecto del AIB sobre la concentración de clorofila b en el fruto de granada roja 'Apaseo tardía'. Las letras iguales indican que no hay diferencias significativas entre tratamientos.

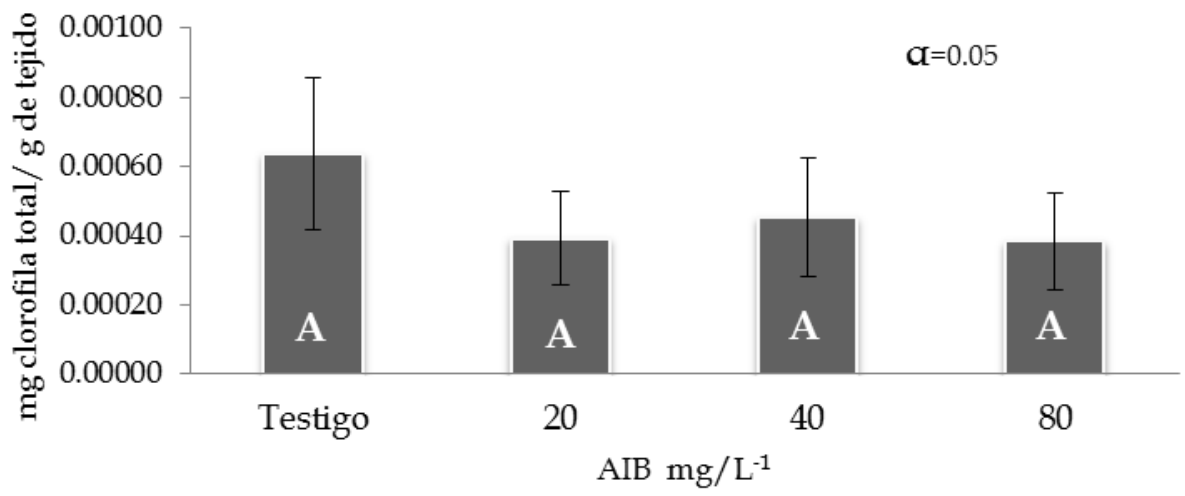

Fig. 6. Efecto del AIB sobre la concentración de clorofila total en el fruto de granada roja 'Apaseo tardía'. Las letras iguales indican que no hay diferencias significativas entre tratamientos. 


\section{DISCUSIÓN}

En esta investigación se observaron diferencias significativas en la conservación de tamaño en longitud del fruto, en donde el AIB aplicado a concentraciones de $20 \mathrm{mg} / \mathrm{L}^{-1}$ tuvo menores pérdidas en comparación con las otras concentraciones. Lo anterior permitiría inferir que esa sería la mejor concentración para la comercialización de granada roja 'Apaseo Tardía' en fresco, pues el tamaño es un aspecto de calidad que, según encuestas realizadas, de manera subjetiva los consumidores consideran de gran importancia, por lo que interviene en la decisión de compra del consumidor, aún por encima de otros aspectos como la marca comercial, su origen o empaque (López, 2003).

Los resultados de pérdida de tamaño en longitud del fruto difieren con los reportados por Velázquez (2017), quien no encontró diferencias significativas en la misma variable al aplicar giberelinas en precosecha; tal vez la diferencia de los resultados se deba al momento de aplicación de la hormona, en precosecha, y en esta investigación los tratamientos se aplicaron en poscosecha y con un regulador de crecimiento diferente. Por otro lado, las giberelinas tienen como principal acción la elongación celular, mientras que las auxinas interfieren en la división celular (Jordán y Casaretto, 2006), lo que también pudo haber influido en la discrepancia de resultados.

Para el resto de las variables evaluadas no se encontraron diferencias significativas, lo que concuerda con los resultados obtenidos por Mercado et al. (2011), quienes realizaron una investigación en poscosecha con frutos de granada roja y al someterlos a una temperatura de $0^{\circ} \mathrm{C}$ no encontraron cambios apreciables en su calidad hasta por 20 días en muestras de frutos intactos; en su trabajo evaluaron tres factores: condición del fruto, agrietado y sin agrietamiento; presentación del producto, arilos libres o gajos; y temperatura de almacenamiento, $0,5,10$ y $20^{\circ} \mathrm{C}$.

En el presente trabajo no se encontraron diferencias estadísticamente significativas en la variable de masa, sin embargo, los frutos tratados con AIB conservaron mayor masa en comparación con el testigo. También se puede apreciar una tendencia en el que a menor concentración de AIB existe menor perdida de la masa del fruto. De acuerdo con Palou et al. (2011), la pérdida de peso se encuentra entre los principales problemas que limitan la frigoconservación de la granada roja. Por otra parte, Mercado et al. (2011), encontraron una disminución estadísticamente significativa al evaluar diferentes temperaturas de almacenamiento del fruto de granada roja con el transcurso del tiempo de la aplicación de los tratamientos, ellos atribuyeron la pérdida de masa a la transferencia de agua al ambiente hasta alcanzar las condiciones de humedad relativa y temperatura previamente fijadas al estar en refrigeración. Es posible, que en este trabajo la aplicación de AIB haya evitado la pérdida de agua.

Los resultados de acidez titulable, que tampoco presentan diferencias significativas, difieren a los obtenidos por Palma et al. (2009), quienes al almacenar arilos de granada roja 'Primosole' durante diez días en atmósferas modificadas de polipropileno notaron un incremento en la acidez titulable del 0.27 al $0.30 \%$. Ellos atribuyeron el incremento de acidez en el jugo de granada roja a la solubilización de $\mathrm{CO}_{2}$ en el tejido. Posiblemente no se encontraron diferencias significativas en este trabajo porque el material experimental fue un fruto completo, a diferencia de autores como Palma et al. (2009), que aplicaron los tratamientos a los arilos; cabe mencionar que estos tratamientos no fueron químicos sino físicos. Otra variante se atribuye al tiempo de experimentación empleado por los autores antes citados que fue de 10 días, mientras que para el presente trabajo fue de 60 días. Según Mercado et al. (2011), en México existe mayor preferencia por el consumo de frutos dulces desplazando en gran medida a los frutos con alto grado de acidez, siguiendo esta afirmación, el tratamiento de $40 \mathrm{mg} / \mathrm{L}^{-1}$ de AIB podría ser el más aceptado por los 
consumidores debido a que el porcentaje de ácido málico es menor.

Los resultados de la presente investigación no mostraron diferencias estadísticamente significativas para la concentración de ácido ascórbico, lo que evidencia una relación directamente proporcional entre la concentración de AIB y ácido ascórbico, es decir, a mayor concentración de la hormona AIB aplicada al fruto, la cantidad de ácido ascórbico en el jugo de granada roja 'Apaseo Tardía' es mayor. Se sabe que la concentración de ácido ascórbico permanece constante durante la maduración en la mayoría de las frutas (Fry, 2010), es factible que la influencia de colectar el material en madurez de consumo haya permitido que la concentración de ácido ascórbico permaneciera similar entre tratamientos al mantenerse estable; Escudero (2016) no observó diferencias significativas entre las concentraciones de ácido ascórbico presentes en el jugo de granada roja 'Apaseo Tardía' que trató con ácido giberélico en precosecha, con la intención de disminuir el número de frutos agrietados. Resultados similares fueron reportados por Velázquez (2017), que tampoco encontró diferencias significativas en la concentración de ácido ascórbico en el jugo de granada roja al aplicar giberelinas en precosecha a sus frutos.

Respecto a la concentración de antocianinas totales, en la presente investigación no se encontraron diferencias significativas entre tratamientos pero se observó una mayor cantidad del pigmento en los frutos tratados con 40 y $80 \mathrm{mg}$ de $\mathrm{AIB} / \mathrm{L}^{1}$ respecto al testigo. Fue evidente que a mayores concentraciones de AIB, el contenido de antocianinas incrementó; el mismo patrón fue observado por Escudero (2016) quien reportó que la concentración de antocianinas aumentó conforme el incremento de la concentración de ácido giberélico aplicado. Al respecto, Legua et al. (2000), reportaron que durante la maduración el contenido de antocianinas totales se incrementó en los cuatro cultivares de granada roja analizados; los autores observaron que el jugo cambió de incoloro a rojo oscuro durante su desarrollo. En el presente trabajo, posiblemente la aplicación de 80 mg de $\mathrm{AIB} / \mathrm{L}^{-1}$ promovió la maduración del fruto, lo que incrementó el contenido de antocianinas, pues características como azúcares totales, acidez, contenido de fenoles y de antocianinas dependen de la madurez de este fruto no climatérico (Palou et al., 2011; Cea, 2011).

En las clorofilas totales, $a$ y $b$ no se encontraron diferencias significativas. Sin embargo, para la clorofila $a$ se observa un comportamiento en el que a menor concentración de AIB aplicado, ésta se encuentra en mayor concentración. La concentración y distribución de los pigmentos presentes en los tejidos son los responsables de la coloración en el epicarpio (Zaccari et al., 2017). Mientras que la clorofila $a$ aporta una coloración verde azulada, la clorofila $b$ da una coloración verde amarillenta (Escudero, 2016). La coloración de los frutos se relaciona con la cultura de consumo, ya que es una de las principales características que se percibe y se asocia con la calidad; por ejemplo, el consumidor puede relacionar el color con la madurez y por consiguiente inferir si posee buen sabor, textura o aroma (López, 2003).

Por todo lo anterior, se puede concluir que el tratamiento de $20 \mathrm{mg} / \mathrm{L}^{-1}$ de AIB presentó diferencias estadísticas significativas al conservar el tamaño del fruto en comparación con los tratamientos de 0,40 y $80 \mathrm{mg} / \mathrm{L}^{-1}$.

En las variables de respuesta masa y tamaño en diámetro del fruto, concentración de antocianinas totales, ácido ascórbico, acidez titulable, clorofila $a, b$ y total, no se presentaron diferencias estadísticamente significativas, por lo que se puede aseverar que las concentraciones de AIB utilizadas en esta investigación no afectaron la calidad del fruto de la granada roja 'Apaseo tardia. 
Por otra parte es importante mencionar que las variables masa, concentración de ácido ascórbico y de antocianinas totales presentan tendencias a responder a la aplicación de AIB, lo que permite inferir la posibilidad de que a mayor tiempo de inmersión en la solución de AIB y de almacenaje de los frutos, las variables de respuesta evaluadas podrían presentar resultados diferentes.

\section{AGRADECIMIENTOS}

El autor principal agradece al laboratorio de Morfofisiología Vegetal de la Facultad de Estudios Superiores Iztacala-UNAM, las facilidades proporcionadas a lo largo de la investigación.

\section{REFERENCIAS}

1. Al-Muammar M.N. y F. Khan, 2012. Obesity: the preventive role of the pomegranate (Punica granatum). Nutrition, 28(6): 595-604. DOI: $\underline{10.1016 / j . n u t .2011 .11 .013}$

2. AOAC (Asociation Oficial Analisys Chemical), 1992. Official methods of analysis of the association of official agricultural chemists, Official method 942.15. American Chemical Society, Washington, D.C.

3. Arias V.C.J. y Toledo H.J., 2000. Manual de manejo poscosecha de frutas tropicales (papaya, piña, plátano y cítricos). FAO, Perú.

4. Aviram M. y L. Dornfeld, 2001. Pomegranate juice consumption inhibits serum angiotensinconverting enzyme activity and reduces systolic blood pressure. Atherosclerosis, 158: 195-198.

5. Bidwell R.G.S., 1993. Fisiología vegetal. AGT editor, S. A. México.

6. Borochov-Neori H., S. Judeinstein, E. Tripler, M. Harari, A. Greenberg, I. Shomer y D. Holland, 2009. Seasonal and cultivar variations in antioxidant and sensory quality of pomegranate (Punica granatum L.) fruit. Journal Food Composition and Analysis, 22(3): 189-195.

DOI: $10.1016 /$ j.jfca.2008.10.011

7. Brackmann A., F.R. Thewes, R.O. Anese, V. Both, W. Linke Jr. y E.E. Schultz, 2015. Aminoethoxyvinylglycine: isolated and combined with other growth regulators on quality of 'Brookfield' apples after storage. Scientia Agricola, 72(3): 221-228.

DOI: 10.1590/0103-9016-2014-0099

8. Cea P.I.A., 2011. Caracterización física, química y sensorial de frutos de granado cv. Wonderful provenientes de tres regiones de Chile (tesis profesional, FCA, Universidad de Chile, Chile).

9. Cohen T., 2011. Pomegranate helps fight heart disease, relieves stress and improves sex life - now it's nature's elixir of youth, claims study http://www.dailymail.co.uk/news/article2060163/Pomegranate-prevents-heart-disease-improves-sex-life-elixir-youth.html

(accesado en febrero 25, 2016).

10. Demerutis C., 2010. Requerimientos de calidad y seguridad en la exportación de frutas y verduras tropicales hacia los mercados mundiales. Revista Iberoamericana Tecnología Poscosecha, 11(1): $1-7$. 
11. El-Ramady H.R., É. Domokos-Szabolcsy, N.A. Abdalla, S.H. Taha y M. Fári, 2015. Postharvest management of fruits and vegetables quality. En: L. Eric (Ed). Sustainable agriculture reviews. Springer Institute of Southeast Asian Studies, Singapore.

12. Escudero M.Y.E., 2016. Efecto del acigigib sobre el agrietamiento y la calidad del fruto de la Granada roja cv. Apaseo tardía (Punica granatum) (Tesis Profesional, FESI, Universidad Nacional Autónoma de México, México).

13. Figueroa C.R., M.C. Opazo, P. Vera, O. Arriagada, M. Díaz y M.A. Moya-Leon, 2012. Effect of postharvest treatment of calcium and auxin on cell wall composition and expression of cell wallmodifying genes in the Chilean strawberry (Fragaria chiloensis) fruit. Food Chemistry, 132(4): 20142022. DOI: $10.1016 / j . f o o d c h e m .2011 .12 .041$

14. Frison E.A. y J. Servinsky (Eds.), 1995. ECP/GR Directory of European Institutions Holding Crop Genetic Resources Collections. Vol 1. IPGRI. Rome, Italy.

15. Fry S.C., 2010. Ripening. En: Thomas B., B. Murray y D. Murphy (Eds). Encyclopedia of applied plant sciences. Elsevier Ltd, The Netherlands.

16. Goldschmidt E.E., 1980. Pigment changes associated with fruit maturation and their control. En: Thimann K.V. (Ed). Senescence in Plants. CRC Press, Boca Raton, Florida.

17. IPGRI (International Plant Genetic Resources Institute), 2001. Regional report CWANA 19992000. IPGRI. Rome, Italy.

18. Jagota. S.K. y H.M. Dani, 1982. A new colorimetic technique for the estimation of vitamina C using Folin phenol Reagent. Analitycal Biochemistry, 127(1): 178-182.

DOI: $10.1016 / 0003-2697(82) 90162-2$

19. Jobling J., R. Pradhan, S.C. Morris, L. Mitchell y A.C. Rath, 2003. The effect of Re Tain plant regulator [(aminoethoyivinylglycine (AVG)] on the postharvest storage life of 'Tegan Blue' plums. Australian Journal of Experimental Agriculture., 43(5): 515-518.

DOI: $\underline{10.1071 / E A 02130}$

20. Jordán, M. y J. Casaretto, 2006. Hormonas y reguladores del crecimiento: Auxinas, giberelinas y citocininas. En: Squeo F.A. y L. Cardemil (Eds), Fisiología vegetal. Ediciones Universidad de La Serena, La Serena, Chile.

21. Kader A.A. (Ed), 2002. Tecnología postcosecha de cultivos hortofrutícolas. UC ANR. Davis, California.

22. Kannangara C. y M. Hansson, 1998. Arrest of chlorophyll accumulation prior to anthocyanin formation in Euphorbia pulcherrima. Plant Physiology and Biochemistry, 36(12): 843-848.

DOI: $\underline{10.1016 / S 0981-9428(99) 80001-1}$

23. Legua P., P. Melgarejo, M. Martínez y F Hernández, 2000. Evolution of anthocyanins content of four pomegranate cultivars (Punica granatum L.) during fruit development. Série A. Séminaires Méditerranéens, 42: 93-97. 
24. López C.A.F., 2003. Manual para la preparación y venta de frutas y hortalizas del campo al mercado. Boletín de servicios agrícolas de la FAO, Roma, Italia.

25. López-Mejía O.A., A. López-Malo y E. Palou, 2010. Granada (Punica granatum L.): una fuente de antioxidantes de interés actual. Temas selectos de ingeniería en alimentos UDLAP, 4(1): 64-73.

26. Mercado S.E., J. Mondragón, L. Rocha y B. Álvarez, 2011. Efectos de condición del fruto y temperatura de almacenamiento en la calidad de granada roja. Revista Mexicana de Ciencias Agrícolas, 3(2): 449-459.

27. Mir M.M., I. Umar, S.A. Mir, M.U. Rehman, G.H. Rather y S.A. Banday, 2012. Quality evaluation of pomegranate crop: a review. International Jornal of Agriculture and Biology, 14(4): 658-667.

28. Palma A., M. Schirra, S. D’Aquino, S. La Malfa, G. Continella, 2009. Chemical properties changes in pomegranate seeds packaged in polypropylene trays. Acta Horticulturae, (818): 323-330.

DOI: $10.17660 /$ ActaHortic.2009.818.48

29. Palou L., C. Montesinos-Herro, V. Tabener, A. Guardado y M.A. del Río, 2011. Enfermedades de poscosecha de la granada en España. Pytoma, (231): 27-32.

30. Peñalver M.M., J.A. López-M., S.A. Streitenberger y O.P. Martínez, 2011. Use of plant extracts as prebiotics, compostions and foods containing such extracts. Patent Number: WO2011036316. http://patentscope.wipo.int/search/en/WO2011036316 (accesado en marzo 02, 2016)

31. Reynolds A.G., D.A. Wardle, C. Zurowski y N.E. Looney, 1992. Phenylureas CPPU and thidiazuron affect yield components, fruit composition, and storage potential of four seedless grape selections. Journal of the American Society for the Horticultural Science, 117(1): 85-89.

DOI: $\underline{10.21273 / J A S H S .117 .1 .85}$

32. SAGARPA (Secretaría de Agricultura, Ganadería, Desarrollo Rural, Pesca y Alimentación), 2015. http://www.sagarpa.gob.mx/saladeprensa/2012/Paginas/2014B602.aspx

(accesado en septiembre 10, 2015).

33. Salveit M.E., 1999. Effect of ethylene on quality of fresh fruits and vegetables. Postharvest Biology and Technology 15(3): 279-292. DOI: $\underline{10.1016 / S 0925-5214(98) 00091-X}$

34. Velázquez P.M.A., 2017. Evaluación del efecto de nitrato de potasio en la brotación de yemas y efecto del ácido giberélico en la calidad del fruto de granada roja (Punica granatum L.) 'Apaseo Tardía' (tesis profesional, FESI, Universidad Nacional Autónoma de México, México).

35. Verma N., A. Mohanty y A. Lal, 2010. Pomegranate genetic resources and germplasm conservation: a review. Fruit, Vegetable and Cereal Science and Biotechnology, 4(2): 120-125. 
36. Whitam F., D. Blaydes y R. Devlin, 1971. Experiments in plant physiology. Van Nostrand Rteinhold Company, New York.

37. Zaccari F., M. del Puerto, B. Vignale y C. Pritsch, 2017. Parámetros colorimétricos y contenido de pigmentos de cinco colores de cáscara de fruto de guayabo [Acca sellowiana (Berg) Burret]. Agrociencia Uruguay, 21(2): 23-30.

BIOCYT Biología, Ciencia y Tecnología, se encuentra actualmente indexada en
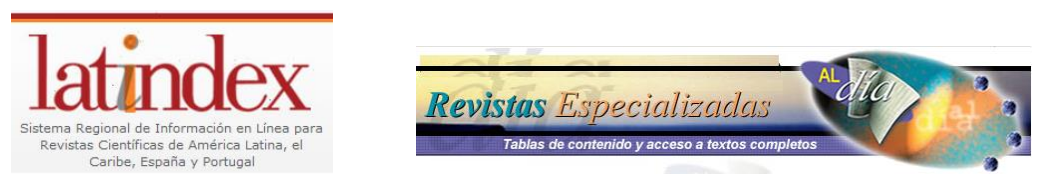

\section{O Dialnet}

\section{Actualidad Iberoamericana}
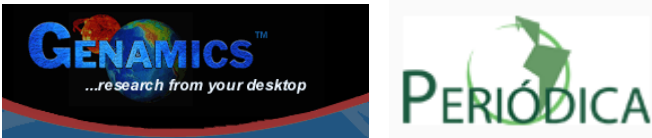

I.1 $\mathrm{s}$ REMERI $\equiv$

INDII $\mathbf{Z E} \mid \begin{aligned} & \text { de Revistas y } \\ & \text { Publicaciones Periódicas Beta }\end{aligned}$

alojada en los repositorios

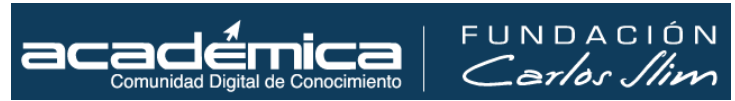

Journals for Iree
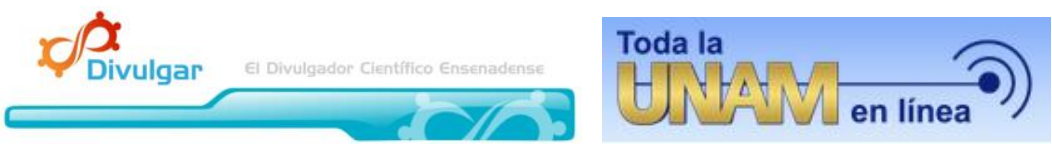

y en bases electrónicas de bibliotecas

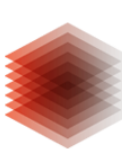

TIB

LEIBNIZ-INFORMATIONSZENTRUM TECHNIK UND NATURWISSENSCHAFTEN UNIVERSITÄTSBIBLIOTHEK 